\section{Incidência de cárie dentária em adolescentes em município do Nordeste brasileiro, 2006}

\author{
Dental caries incidence in adolescents in a city \\ Northeast Brazil, 2006
}

\footnotetext{
${ }^{1}$ Centro de Ciências da Saúde, Universidade de Fortaleza, Fortaleza, Brasil. 2 Departamento de Odontologia, Universidade Federal do Rio Grande do Norte, Natal, Brasil. 3 Secretaria de Estado da Saúde do Ceará, Fortaleza, Brasil.

4 Programa de Pós-graduação em Ciências da Saúde, Universidade Federal do Rio Grande do Norte, Natal, Brasil.

Correspondência L. R. A. Noro Assessoria Pedagógica, Centro de Ciências da Saúde, Universidade de Fortaleza. $A v$. Washington Soares 1321, Fortaleza, CE 60811-905, Brasil. noro@unifor.br
}

\begin{abstract}
The main objective of this study was to evaluate the incidence of tooth decay in adolescents, associated with socioeconomic status, access to services, and self-perceived oral health. This was a longitudinal study using an epidemiological survey of dental caries and a structured questionnaire with a sample of 688 adolescents residing in Sobral, Ceará State, Brazil. Incidence increased progressively with age, from 1.60 at 12 years of age to 2.28 at 15, with a mean incidence of 1.86 decayed teeth per adolescent. Among the study variables, tooth pain in the previous six months $[R R=1.46$ (1.22-1.76)], school lunch [RR = 1.45 (1.21-1.74)], frequency of dental appointments $[R R=1.48$ (1.33-1.79)], and access to the Health Service [RR = 1.21 (1.01-1.45)], adjusted by perceived need for treatment, were associated with high caries incidence. It is essential for health professionals and health system managers to formulate public policies that are not limited merely to clinical and preventive aspects, encouraging the population to struggle for better living conditions and allowing equitable access to services and developing collective management of health actions.
\end{abstract}

Dental Caries; Adolescent; Oral Health
Luiz Roberto Augusto Noro 1 Angelo Giuseppe Roncalli 2

Francisco Ivan Rodrigues Mendes Júnior ${ }^{3}$ Kenio Costa Lima 4

\section{Introdução}

Aproximadamente $70 \%$ dos países do mundo atingiram a meta estabelecida pela Organização Mundial da Saúde (OMS): CPOD $\leq 3$ aos 12 anos de idade, desde que este foi proposto 1 . Da mesma forma, isso aconteceu com a população brasileira. Entretanto, apesar das sinalizações de melhoria na situação da cárie dentária ${ }^{2}$, a doença ainda é um dos agravos de maior prevalência em nosso meio. Segundo o relatório do Projeto SB Brasil 2003 3, em relação à cárie, o Brasil atingiu as metas da OMS para o ano 2000 somente para a idade de 12 anos, e, em parte, isso aconteceu devido às crianças das regiões Sul e Sudeste.

Em pesquisas sobre cárie dentária, uma variável dependente importante é a incidência - que pode ser avaliada como a diferença entre a prevalência de cárie no início do estudo e a prevalência ao final da pesquisa 4,5 . Porém, poucos são os estudos em Epidemiologia em saúde bucal que analisam mudanças ao longo do tempo 6,7. A incidência das doenças é um dos principais instrumentos para avaliação da condição de saúde de uma determinada população e deve orientar o planejamento de ações que venham a contribuir para uma melhora significativa, em especial nos serviços de saúde públicos.

O modelo de atenção à saúde no Município de Sobral (Ceará) foi organizado a partir de 1997 com base nos princípios e diretrizes do Sistema Único de Saúde, com base em ações que não 
se limitavam à atuação do setor saúde, requerendo o exercício de práticas interdisciplinares e multiprofissionais, ancorado em estratégias como a saúde da família ${ }^{8}$. A inclusão de equipes de saúde bucal na Estratégia Saúde da Família ocorreu gradualmente a partir de 1998, tendo como desafio trabalhar em uma nova perspectiva de atuação, com foco na promoção da saúde, numa prática mais coletiva e participativa do profissional de saúde bucal 9. Deve-se ressaltar que esta quebra de paradigma somente pode ser viabilizada no enfrentamento da grande demanda reprimida para ações curativas, mediante informações epidemiológicas que possam efetivamente provocar um novo direcionamento das ações de saúde bucal.

O objetivo deste trabalho foi avaliar a incidência da cárie dentária na dentição permanente entre os anos de 2000 a 2006 em adolescentes de 11 a 15 anos de idade no Município de Sobral e sua associação a fatores relativos à condição sócio-econômica, acesso a ações e serviços e autopercepção, considerando que para viabilizar a proposta a ser implantada pelo município havia a necessidade de um maior conhecimento da realidade epidemiológica da população em relação à cárie dentária.

\section{Métodos}

O presente estudo desenvolveu-se com delineamento do tipo longitudinal, caracterizado enquanto estudo analítico observacional por meio de exames bucais para identificação da incidência de cárie dentária na população adolescente (11 a 15 anos de idade) do Município de Sobral.

O referencial inicial para desenvolvimento do estudo foi a pesquisa Condições de Saúde das Crianças no Município de Sobral - Ceará, realizada durante os anos de 1999 a 2000. Para esta pesquisa, a amostra foi composta por sorteio, pelo cadastro de domicílios elaborado por profissionais de saúde da Secretaria de Desenvolvimento Social e Saúde de Sobral, que incluía as crianças residentes na área urbana nascidas entre 1990 e 1994 estratificadas pelo ano de nascimento. Para o cálculo da amostra deste estudo foi considerada uma população estimada de 18.668 crianças na faixa etária de 5 a 9 anos de idade (Instituto Brasileiro de Geografia e Estatística. Censo demográfico - 1996. http:/ /www. ibge.gov.br). Tomando-se como referência uma prevalência de $10 \%$ entre as diversas situações previstas no questionário 10 e considerando uma margem de erro de $10 \%$ e um nível de $95 \%$ de confiança, o número final encontrado, ajustado para populações finitas, foi de 2.900 crianças.
Mas, considerando-se a capacidade operacional disponível para a pesquisa, a amostra final foi composta de 3.425 crianças. Nesta fase da pesquisa, foram realizadas entrevistas domiciliares com as mães das crianças, visando à aplicação de questionário semi-estruturado.

Entre estas 3.245 crianças foram sorteadas 1.021 crianças que compuseram a amostra do levantamento epidemiológico de cárie, condição gengival e má oclusão 11 , realizado como parte integrante desta pesquisa. Considerandose uma prevalência de $30 \%$, margem de erro de $10 \%$ e nível de $95 \%$ de confiança, o tamanho da amostra seria de 710 crianças. Todavia, como se previa uma continuidade da pesquisa na perspectiva de um estudo longitudinal, optou-se por trabalhar com a amostra de 1.021 crianças.

Enfim, para composição da amostra do seguimento do estudo em 2006 foi feita a busca ativa dos 1.021 indivíduos residentes na zona urbana do Município de Sobral que haviam sido examinados no ano de 2000, à época com idade entre 5 e 9 anos. Os examinadores visitaram todos os domicílios tendo como base a ficha de exame realizado preliminarmente e os critérios de exclusão para participação na pesquisa foram mudança de endereço ou domicílio fechado após três tentativas de localização. Não aconteceram recusas em participar da pesquisa.

Após as visitas, 688 adolescentes foram examinados e seus responsáveis responderam ao questionário com perguntas relativas à condição sócio-econômica, acesso a ações e serviços e percepção auto-referida.

Previamente ao levantamento, os pesquisadores participaram de capacitação técnica para definição dos padrões a serem estabelecidos quando da realização dos exames e aplicação dos questionários. Após a capacitação, foi realizada calibração da qual, em um primeiro momento, participaram 15 cirurgiões-dentistas. Esta fase serviu para observação da consistência intra-examinadores e dela foram selecionados os pesquisadores que apresentaram, nesta etapa, concordância intra-examinador maior que 95\%. Na segunda fase, foi realizada a calibração pela técnica do consenso entre os examinadores observando-se uma concordância geral interexaminadores de 0,92 a 0,97 com coeficiente kappa variando de 0,86 a 0,96 , demonstrando excelente grau de concordância.

A coleta de dados foi realizada com sete equipes compostas por sete cirurgiões-dentistas (examinadores) e sete auxiliares de consultório dentário (anotadoras) da Secretaria Municipal de Saúde de Sobral. Os exames foram realizados no próprio domicílio, sendo a visita da equipe 
previamente agendada pelo agente comunitário de saúde responsável pela área.

Para realização dos exames, foi construída ficha específica com emprego dos códigos e critérios preconizados pela OMS 12 para levantamentos epidemiológicos em saúde bucal.

Cada participante do estudo recebeu uma escova de dente para realização da higiene bucal anteriormente ao exame bucal, sob orientação da auxiliar de consultório dentário, sendo este fator indispensável à padronização de todos os exames. Após o levantamento epidemiológico, foi aplicado questionário para os responsáveis pelo adolescente examinado, na grande maioria das vezes representada pela mãe do adolescente.

A variável dependente do estudo foi a incidência de cárie, medida pela experiência de nova cárie por dente em um indivíduo entre o período do primeiro exame realizado no ano 2000 e o último exame realizado em 2006, conforme proposto em estudo de Kallestal \& Stenlund 13.

Assim, a incidência foi considerada positiva nas situações em que o dente encontrava-se hígido no primeiro exame e passou a apresentar a situação "cariado", "extraído", "com extração indicada" ou "restaurado" no segundo exame. Também entraram nesta categoria os dentes restaurados no primeiro exame e que apresentaram a situação "cariado", "extraído" ou "com extração indicada" no segundo exame. Além disto, todos os dentes permanentes que não estavam presentes no primeiro exame e que apresentaram a situação "cariado", "extraído", "com extração indicada" ou "restaurado" no segundo exame também foram computados como dentes com incidência positiva de cárie.

Utilizou-se o programa Epi Info versão 6.04 (Centers for Disease Control and Prevention, Atlanta, Estados Unidos) para construção do banco de dados e a análise estatística foi feita com uso do programa SPSS for Windows, versão 13.0 (SPSS Inc., Chicago, Estados Unidos). Para avaliar a relação entre as variáveis independentes e a incidência de cárie, realizou-se teste de associação pelo qui-quadrado e se estimou o risco relativo (RR) e seu respectivo intervalo de confiança.

As variáveis independentes, classificadas nas categorias "condição sócio-econômica", "acesso a ações e serviços" e "autopercepção", foram dicotomizadas em situações adequadas e inadequadas e avaliadas mediante análise univariada. As variáveis independentes com nível de significância $\mathrm{p}<0,20$ compuseram a análise multivariada utilizando-se a regressão de Poisson. No modelo final somente foram mantidas as variáveis independentes que, após a regressão, apresentaram-se estatisticamente significativa, ou seja, $\mathrm{p}<0,05$.
O trabalho foi previamente submetido e aprovado sob o número 218 pelo Comitê de Ética em Pesquisa da Universidade Estadual Vale do Acaraú, garantindo meios adequados de permitir máxima segurança aos participantes da pesquisa, sempre respeitando os princípios do anonimato e privacidade.

\section{Resultados}

O presente estudo foi realizado com um total de 688 participantes $(48,8 \%$ do sexo feminino e $51,2 \%$ do sexo masculino) entre 11 e 15 anos de idade, residentes no Município de Sobral. A distribuição nas idades foi de 18,3\% com 11 anos, 19,9\% com 12 anos, 18,6\% com 13 anos, 19,1\% com 14 anos e 24,1\% com 15 anos. Em relação à linha base da coorte, a taxa de resposta do presente estudo foi de $67,4 \%$, acontecendo a grande maioria das perdas em virtude de mudança de residência do adolescente e recusas em participar da pesquisa.

A partir da análise, verificou-se uma incidência de cárie que variou de 1,95 dente $(\mathrm{DP}=1,98)$ por indivíduo aos 11 anos até 2,28 dentes (DP = $2,45)$ por indivíduo aos 15 anos, com uma incidência média de 1,86 dente ( $\mathrm{DP}=2,15$ ), conforme pode ser observado na Figura 1.

A incidência aumentou progressivamente com a idade passando de 1,60 (DP $=1,97$ ) nos adolescentes com 12 anos de idade para atingir 2,28 ( $\mathrm{DP}=2,45)$ para aqueles com 15 anos.

Pela análise das variáveis independentes, constatou-se que $97,4 \%$ dos participantes eram estudantes, e, deste grupo, 89,1\% estudavam em escola pública. Do total da amostra, $84,9 \%$ tinham moradia própria. Dos que tiveram acesso ao serviço odontológico, $87,1 \%$ o classificaram como bom ou ótimo. Com relação à percepção de saúde bucal, $82,8 \%$ avaliaram sua mastigação como boa ou ótima, assim como 95\% em relação à fala. Em relação aos produtos para prevenção, a escova de dente e o dentifrício foram praticamente de uso universal (98,4\% e 99,6\%, respectivamente), em contraposição ao fio dental que foi utilizado por apenas $8,1 \%$ dos participantes da pesquisa. $\mathrm{O}$ abastecimento público de água tratada e fluoretada atinge $98,7 \%$ desta população.

As variáveis pessoas por cômodo, escolaridade do chefe da família, renda familiar, renda do chefe da família, desnutrição, característica do domicílio, coleta de lixo, local de atendimento, falta de atendimento pelo serviço, local onde não foi atendido, realização de ações coletivas, orientação sobre prevenção, local de recebimento de orientação, quem orienta, freqüên- 


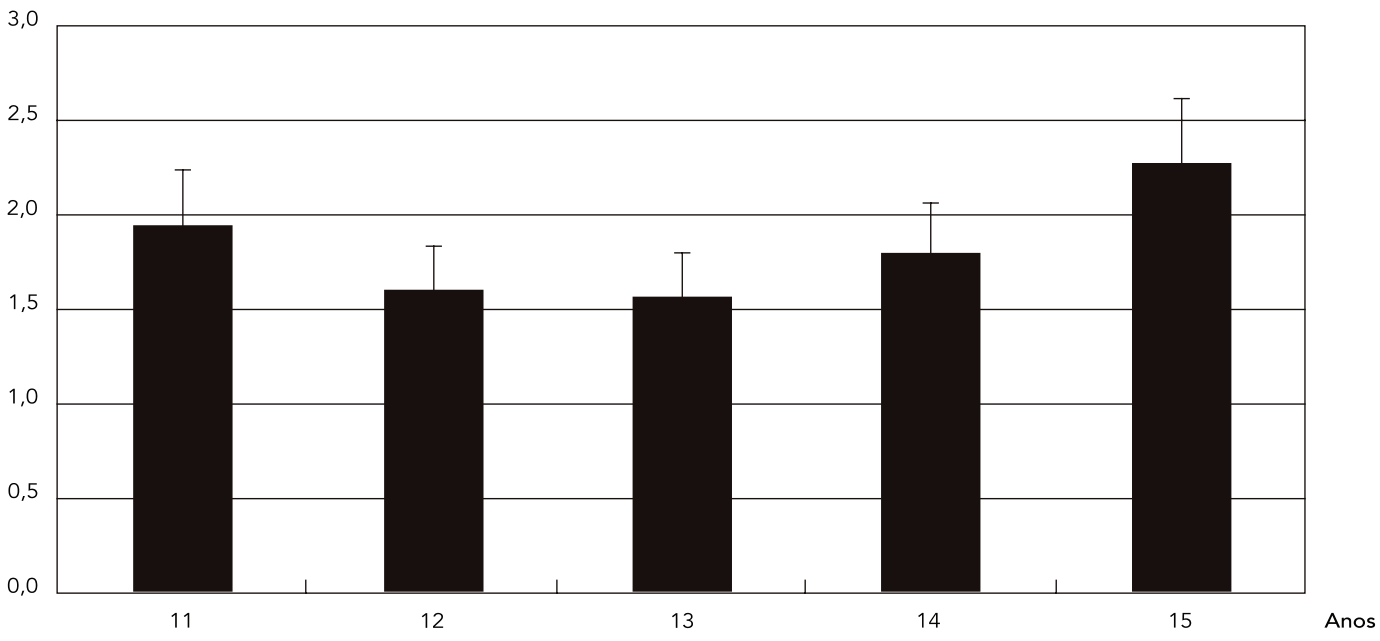

Tamanhos da amostra: 11 anos $=126 ; 12$ anos $=137 ; 13$ anos $=128 ; 14$ anos $=131 ; 15$ anos $=166$

cia de escovação, condição da escova, visita do agente comunitário da saúde (ACS), cadastro no Programa Saúde da Família (PSF), percepção sobre aparência e percepção na relação com pessoas não apresentaram significância, e, portanto, não entraram no modelo de regressão.

As demais variáveis independentes, que apresentaram $\mathrm{p}<0,20$, foram incluídas na regressão de Poisson e sua distribuição em relação à incidência, considerando-se o qui-quadrado, valor de "p", o RR, o valor de "p" ajustado e o RR ajustado encontram-se na Tabela 1.

Pode-se observar que as variáveis que demonstraram relação com a incidência de cárie foram "dor de dente nos últimos seis meses", apresentando RR = 1,46 (IC95\%: 1,22-1,76); a "merenda escolar", apontando que aqueles que consumiam merenda escolar sempre durante a infância (5 a 9 anos de idade) apresentaram maior risco $[\mathrm{RR}=1,45$ (IC95\%: 1,21-1,74)] de apresentar alta incidência, quando comparados àqueles que nunca comiam ou a comiam eventualmente; a "freqüência de consulta ao dentista" com intervalo menor que um ano apresentou um risco 1,48 (IC95\%: 1,23-1,79) maior do que aqueles que realizaram consulta com intervalos superiores a um ano, assim como aqueles que têm "acesso pontual à unidade básica de saúde" apresentaram maior risco de alta incidência de cárie [RR = 1,21 (IC95\%: 1,01-1,45)] quando comparados àqueles cujo acesso é regular.

\section{Discussão}

O presente estudo procurou preencher uma grande lacuna na área da Epidemiologia em saúde bucal, uma vez que, de acordo com Peres et al. ${ }^{14}$, existem poucos estudos epidemiológicos de coorte, de base populacional, que investigaram tais aspectos. Por isso mesmo, é grande a dificuldade em comparar os achados deste estudo com outros da mesma natureza.

Outro elemento importante a ser destacado é relativo ao acompanhamento do segmento do estudo, já que a presente pesquisa apresentou uma perda de $32,62 \%$ em relação à linha de base da coorte, o que pode ter provocado algumas distorções nas estimativas de incidência, bem como nas associações encontradas.

Em estudo de Karger 15, observa-se uma incidência de cárie na faixa etária de 12 a 15 anos de idade 4,3 maior que o encontrado em Sobral $(1,86)$. Deve-se ressaltar, entretanto, que naquele estudo ${ }^{15}$ o referencial usado foi a face do dente.

A alta incidência de cárie observada na idade de 11 anos, em relação às subseqüentes, deve estar relacionada ao fato de que a maioria das crianças com cinco anos de idade, no primeiro estudo, não apresentava a erupção do primeiro molar permanente e, desta forma, todo o acúmulo de novas experiências de cárie neste dente foram percebidas na idade de 11 anos. 
Associação entre o desfecho "incidência de cárie" e as variáveis independentes utilizadas no estudo. Sobral, Ceará, Brasil, 2006.

\begin{tabular}{|c|c|c|c|c|c|c|c|}
\hline \multirow[t]{2}{*}{ Variável } & \multicolumn{3}{|c|}{ Incidência de cárie } & \multirow[t]{2}{*}{ Valor de $p$} & \multirow[t]{2}{*}{ RR (IC95\%) } & \multirow{2}{*}{$\begin{array}{c}\text { Valor de p } \\
\text { ajustado }\end{array}$} & \multirow{2}{*}{$\begin{array}{l}\text { RR ajustado } \\
\text { (IC95\%) }\end{array}$} \\
\hline & $\mathbf{n}$ & Média & DP & & & & \\
\hline \multicolumn{8}{|c|}{ Dor de dente nos últimos seis meses } \\
\hline Ausente & 467 & 1,46 & 1,86 & $<0,001$ & $1,84(1,56-2,17)$ & $<0,001$ & $1,46(1,22-1,76)$ \\
\hline Presente & 218 & 2,68 & 2,41 & & & & \\
\hline Total & 685 & 1,85 & 2,13 & & & & \\
\hline \multicolumn{8}{|l|}{ Morbidade referida } \\
\hline Não & 223 & 1,28 & 1,76 & $<0,001$ & $1,67(1,36-2,05)$ & $<0,001$ & $1,71(1,36-2,14)$ \\
\hline Sim & 463 & 2,14 & 2,26 & & & & \\
\hline Total & 686 & 1,86 & 2,15 & & & & \\
\hline \multicolumn{8}{|c|}{ Freqüência de consulta ao dentista } \\
\hline 1 ano e mais & 307 & 1,61 & 2,16 & $<0,001$ & $1,42(1,18-1,71)$ & $<0,001$ & $1,48(1,23-1,79)$ \\
\hline Menos de 1 ano & 301 & 2,29 & 2,16 & & & & \\
\hline Total & 608 & 1,95 & 2,19 & & & & \\
\hline \multicolumn{8}{|c|}{ Acesso à Unidade Básica de Saúde } \\
\hline Sempre & 267 & 1,69 & 1,98 & 0,086 & $1,17(0,98-1,39)$ & 0,035 & $1,21(1,01-1,45)$ \\
\hline Às vezes/Nunca & 418 & 1,97 & 2,25 & & & & \\
\hline Total & 685 & 1,86 & 2,15 & & & & \\
\hline \multicolumn{8}{|l|}{ Merenda escolar } \\
\hline Às vezes/Nunca & 270 & 1,48 & 1,90 & $<0,001$ & $1,44(1,19-1,73)$ & $<0,001$ & $1,45(1,21-1,74)$ \\
\hline Sempre & 345 & 2,13 & 2,24 & & & & \\
\hline Total & 615 & 1,84 & 2,12 & & & & \\
\hline \multicolumn{8}{|l|}{ Renda da mãe } \\
\hline Sem renda & 364 & 1,74 & 2,08 & 0,108 & $1,15(0,97-1,38)$ & & \\
\hline Com renda & 277 & 2,01 & 2,16 & & & & \\
\hline Total & 641 & 1,86 & 2,12 & & & & \\
\hline \multicolumn{8}{|c|}{ Escolaridade da mãe (anos) } \\
\hline 8 e mais & 335 & 1,67 & 1,98 & 0,003 & $1,21(1,02-1,45)$ & & \\
\hline Menos de 8 & 322 & 2,03 & 2,26 & & & & \\
\hline Total & 657 & 1,85 & 2,13 & & & & \\
\hline \multicolumn{8}{|l|}{ Consulta ao dentista } \\
\hline Sim & 611 & 1,95 & 2,19 & 0,003 & $0,608(0,44-0,85)$ & & \\
\hline Não & 76 & 1,18 & 1,69 & & & & \\
\hline Total & 687 & 1,86 & 2,15 & & & & \\
\hline \multicolumn{8}{|c|}{ Como classifica sua saúde bucal } \\
\hline Favorável & 300 & 1,54 & 2,05 & 0,001 & $1,36(1,13-1,63)$ & & \\
\hline Desfavorável & 363 & 2,09 & 2,17 & & & & \\
\hline Total & 663 & 1,84 & 2,13 & & & & \\
\hline \multicolumn{8}{|c|}{ Recebimento da escova } \\
\hline Recebe & 426 & 1,72 & 2,06 & 0,022 & $1,22(1,03-1,46)$ & & \\
\hline Não recebe & 258 & 2,11 & 2,28 & & & & \\
\hline Total & 684 & 1,87 & 2,15 & & & & \\
\hline
\end{tabular}

DP: desvio-padrão; RR: risco relativo; IC95\%: intervalo de 95\% de confiança.

Tendo em conta os resultados do primeiro levantamento epidemiológico realizado em Sobral no ano 2000, quando estes adolescentes estavam na faixa etária de 5 a 11 anos 11, podese constatar que a projeção do CPOD para esta população aos 12 anos de idade é igual a 1,80, ou seja, apresenta-se de forma bastante satisfató- ria, tendo-se como referência as metas da OMS para o ano 2000 16. Se compararmos estes dados com os encontrados no último levantamento epidemiológico realizado em 2003 no Brasil 3, observa-se uma maior adequação deste índice para a população sobralense na idade de 12 anos do que a da população brasileira (CPOD = 
$2,78)$ e da população nordestina $(\mathrm{CPOD}=3,19)$. Ressalte-se que o município apresenta a água de abastecimento público fluoretada desde 1971, o que pode ser um dos fatores que explica a baixa incidência de cárie nesta faixa etária. Contudo, há necessidade de intervenção mais eficiente dos serviços de saúde, pois se verificou um incremento na incidência de cárie com a idade.

Em relação às variáveis independentes que não apresentaram relação com a incidência de cárie é importante destacar que o alto número de escovações por dia (3 vezes ou mais), quando comparado a um número menor (1 a 2 vezes) não apresentou significância, ou seja, aparentemente, o importante é que a escovação seja realizada. Tais dados, entretanto, conflitam-se com os encontrados por Smyth \& Caamaño 17, para os quais a incidência de cárie estaria diretamente relacionada à baixa freqüência de escovação, além do alto consumo de doces.

Quanto à variável "quem orienta medidas educativas", tão citada nas atividades de promoção de saúde, encontrou-se neste estudo, como em outros 18,19, que estas orientações, prestadas por profissionais da área de saúde bucal, não apresentaram influência na baixa incidência de cárie. Considerando-se o potencial da educação, cabe uma reflexão se o posicionamento dos profissionais de saúde adotado na maioria das "palestras educativas" seria a grande limitação no impacto que medidas como estas poderiam significar.

$\mathrm{Na}$ análise do atual estudo, observou-se que das variáveis independentes definidas preliminarmente como "condição sócio-econômica" o consumo de merenda escolar destes adolescentes, quando os mesmos freqüentaram a escola na infância, foi de grande relevância. Constatouse alta incidência de cárie naqueles que consumiam a merenda sempre. A variável merenda tem um componente biológico relacionado à condição da merenda (composição dietética), assim como um componente sócio-econômico porque muitas crianças têm na merenda escolar sua principal refeição. Tais achados remetem à necessidade de uma verificação dos alimentos constantes desta merenda, visto que seu consumo esteve fortemente relacionado à alta incidência. De acordo com Peres et al. 20, hábitos de consumo de produtos cariogênicos, sobretudo com relação à sua freqüência, parece ser o fator comportamental mais comprovadamente aceito no desenvolvimento do processo carioso. Como o consumo da merenda é praticamente exclusivo em escolas públicas, os atuais resultados podem remeter à similaridade entre os dados achados neste estudo e o observado por Maltz \& Silva 21 e em Hoffmann et al. 22 que identificaram maior prevalência de cárie em alunos da rede pública, quando comparados com os de escola privada.

Neste ponto, entende-se que a visão de Källestål \& Wall 23 de que as condições sócio-econômicas não são um fator em si, mas que afetam a incidência de cárie por meio de outras variáveis é perfeitamente compatível com os resultados deste estudo. Concorda-se com estes autores que o achado das variáveis é a chave para se entender como devem ser oferecidas melhores estratégias para a prevenção da cárie.

Das variáveis independentes relativas às ações e serviços, aquela que apresentou associação positiva com o aumento da incidência de cárie foi a dor de dente. Neste estudo, crianças que apresentaram dor de dente tinham um risco maior de alta incidência que as que procuraram atendimento odontológico por outro motivo. Esta situação, nos dias de hoje, representa a total falta de acesso tanto a medidas preventivas como a baixa resolubilidade dos serviços de saúde, quer privados, pelo exclusivo direcionamento a camadas mais abastadas da população no caso dos consultórios particulares, ou a quem pode ao menos pagar um plano de saúde odontológico, representado na maioria das vezes por prestação de assistência com duvidosa qualidade; quer públicos, pela limitação no acesso, fato que resulta em baixa resolubilidade. Vale ressaltar que nesta faixa etária a grande maioria da dor de dente é provocada pela cárie, processo infecto-contagioso de lenta progressão que só leva à dor quando nenhuma medida primária de atenção à saúde bucal foi tomada.

Deve-se considerar que o acesso ao serviço odontológico não pode ser interpretado como uma mera garantia à assistência odontológica, medida que segundo Nadanovsky \& Sheiham 24 não tem representado melhoria no perfil epidemiológico em diversos países europeus. O presente estudo indica tal situação de forma bastante clara, pois aqueles que utilizaram o serviço odontológico em intervalo menor que um ano foram os que apresentaram maior risco de alta incidência de cárie. Logo, a visita regular ao serviço odontológico, tida como eficiente na prevenção de doenças bucais, na realidade tem servido para que pacientes com maior acúmulo de necessidades resolvam clinicamente estes problemas.

$\mathrm{O}$ acesso aos serviços de saúde deve ser pensado na perspectiva de um serviço humanizado, desenvolvido por uma equipe de saúde bucal efetivamente vinculada aos interesses da população, que tenha como grande objetivo proporcionar, junto com os gestores dos serviços públicos de saúde, condições adequadas para melhoria das condições de saúde bucal não apenas por intermédio de medidas individuais. Tais medi- 
das devem ser direcionadas para a redução da grande desigualdade social, apontada por vários autores como o principal fator na determinação da maioria dos agravos em saúde bucal, em especial a cárie.

Outra variável que se comportou de forma bastante interessante no presente estudo foi o acesso à unidade básica de saúde. Aqueles que têm acesso regular apresentaram menor risco de alta incidência de cárie, o que pode indicar uma preocupação dos profissionais destas unidades de saúde com medidas preventivas, em especial as coletivas, uma vez que no Município de Sobral a cobertura da Estratégia Saúde da Família com equipe de saúde bucal é praticamente universal. Corroborando isto, Antunes et al. 25 observam em seu estudo que cidades mais ricas apresentam maior número de profissionais de saúde bucal e menor prevalência de cárie, destacando a ne- cessidade de se repensar o papel da atenção em saúde bucal.

Por isto mesmo, concorda-se com Baldani et al. 26: há necessidade da implementação de medidas e políticas compensatórias para amenizar os efeitos danosos das desigualdades sociais porque que elas se tornam mais graves se estratégias preventivas não são capazes de prover ao menos benefícios equivalentes para grupos de baixo nível sócio-econômico.

Entretanto, é fundamental que gestores e profissionais de saúde formulem políticas públicas que não se restrinjam aos aspectos classicamente identificados como "área da saúde" (atendimento clínico e prevenção de doenças), estimulando a população na luta pela conquista por melhores condições de vida, viabilizando eqüidade no acesso aos serviços e desenvolvendo uma gestão coletiva das ações de saúde.

\section{Resumo}

O presente estudo teve como objetivo analisar a incidência da cárie dentária em adolescentes associada à condição sócio-econômica, acesso a serviços e autopercepção. É um estudo longitudinal por meio de levantamento epidemiológico de cárie e questionário estruturado com amostra composta por 688 adolescentes residentes no Município de Sobral, Ceará, Brasil. A incidência aumentou progressivamente com a idade, passando de 1,60 nos adolescentes com 12 anos até 2,28 para os com 15 anos, registrando-se uma incidência média de 1,86 dentes cariados por adolescente. Entre as variáveis estudadas, dor de dente nos últimos seis meses $[R R=1,46(1,22-1,76)]$, merenda escolar $[R R=1,45(1,21-1,74)]$, freqüencia de consulta ao dentista $[R R=1,48(1,33-1,79)]$ e acesso à unidade de saúde $[R R=1,21(1,01-1,45)]$, ajustadas por morbidade referida apresentaram relação com a alta incidência de cárie. É fundamental que gestores e profissionais de saúde formulem políticas públicas que não se restrinjam a aspectos como atendimento clínico e prevenção de doenças, estimulando a população na luta pela conquista por melhores condições de vida, viabilizando eqüidade no acesso aos serviços e desenvolvendo uma gestão coletiva das ações de saúde.

Cárie Dentária; Adolescente; Saúde Bucal

\section{Colaboradores}

L. R. A. Noro e A. G. Roncalli fizeram a revisão sistemática da literatura e participaram de todas as etapas de elaboração do artigo. F. I. R. Mendes Júnior participou da elaboração do instrumento, acompanhou e coordenou a coleta de dados e colaborou na redação final do artigo. K. C. Lima colaborou na revisão sistemática da literatura, participou da discussão dos resultados e da redação final do artigo. 


\section{Referências}

1. Nishi M, Stjernswärd J, Carlsson P, Bratthall D. Caries experience of some countries and areas expressed by the Significant Caries Index. Community Dent Oral Epidemiol 2002; 30:296-301.

2. Coordenação de Saúde Bucal, Departamento de Assistência e Promoção à Saúde, Secretaria de Assistência à Saúde, Ministério da Saúde. Levantamento epidemiológico em saúde bucal: 1a etapa - cárie dental - projeto. Brasília: Ministério da Saúde; 1996.

3. Departamento de Atenção Básica, Secretaria de Atenção à Saúde, Ministério da Saúde. Projeto SB Brasil 2003. Condições de saúde bucal da população brasileira 2002-2003: resultados principais. Brasília: Ministério da Saúde; 2004.

4. Ekstrand KR. Improving clinical visual detection potencial for caries clinical trials. J Dent Res 2004; 83:67-71.

5. Mancl LA, Hujoel PP, DeRouen TA. Efficiency issues among statistical methods for demonstrating efficacy of caries prevention. J Dent Res 2004; 83:95-8.

6. Holst D, Schuller AA. Oral health changes in an adult Norwegian population: a cohort analytical approach. Community Dent Oral Epidemiol 2000; 28:102-11.

7. Broadbent JM, Thomson WM. For debate: problems with DMF index pertinent to dental caries data analysis. Community Dent Oral Epidemiol 2005; 33:400-9.

8. Andrade LOM, Barreto ICHC, Goya N, Martins Júnior T. Organização da atenção básica e estratégia saúde da família em Sobral-Ceará-Brasil: principais avanços e desafios na perspectiva de produzir mudanças positivas na saúde. In: Suassuna A, Fernandes J, Seclen-Palacin A, organizadores. Experiências e desafios da atenção básica e saúde familiar: caso Brasil. Brasília: Organização Pan-Americana da Saúde; 2004. p. 143-79.

9. Teixeira AH, Bezerra MM, Pinto VPT. Saúde bucal na estratégia Saúde da Família: conhecendo a atuação das equipes em Sobral, CE. SANARE 2005; 6:9-18.

10. Barreto ICHC. Morbidade referida e seus condicionantes em crianças de 5 a 9 anos da zona urbana de Sobral-CE [Tese de Doutorado]. São Paulo: Faculdade de Medicina, Universidade de São Paulo; 2006.

11. Noro LRA, Oliveira AGRC, Mendes Júnior FIR, Lima KC. Children oral health status from Sobral-Ceará. Stoma 2008; 88:4-8.

12. Organização Mundial da Saúde. Levantamentos básicos em saúde bucal. 4a Ed. São Paulo: Editora Santos; 1999.
13. Källestål C, Stenlund H. Different analytical approaches in an experimental cohort study on preventive measures for caries in adolescents: a comparison between incidence density and increment analysis. Caries Res 2003; 37:44-50.

14. Peres MA, Latorre MRDO, Sheiham A, Peres KG, Barros FC, Hernandez PG, et al. Determinantes sociais e biológicos da cárie dentária em crianças de 6 anos de idade: um estudo transversal aninhado numa coorte de nascidos vivos no Sul do Brasil. Rev Bras Epidemiol 2003; 6:293-306.

15. Karger AG. Caries incidence and lesion progression from adolescence. Caries Res 2004; 38:130-41.

16. Federation Dentaire Internationale. Global goals for oral health in the year 2000. Int Dent J 1982; 32:74-7.

17. Smyth E, Caamaño F. Factors related to dental health in 12-years-old children: a cross-sectional study in pupils. Gac Sanit 2005; 19:113-9.

18. Kay L, Locker D. Is dental health education effective? A systematic review of current evidence. Community Dent Oral Epidemiol 1996; 24:231-5.

19. Vanobbergen J, Declerck D, Mwalili S, Martens L. The effectiveness of a 6-year oral health education programme for primary schoolchildren. Community Dent Oral Epidemiol 2004; 32:173-82.

20. Peres KGA, Bastos JRM, Latorre MRD. Severidade de cárie em crianças e relação com aspectos sociais e comportamentais. Rev Saúde Pública 2000; 34:402-8.

21. Maltz M, Silva BB. Relação entre cárie, gengivite e fluorose e nível socioeconômico em escolares. Rev Saúde Pública 2001; 35:170-6.

22. Hoffmann RHS, Cypriano S, Sousa MLR, Wada RS Experiência de cárie dentária em crianças de escolas públicas e privadas de um município com água fluoretada. Cad Saúde Pública 2004; 20:522-8.

23. Källestål C, Wall S. Socio-economic effect on caries. Incidence data among Swedish 12-14-year-olds. Community Dent Oral Epidemiol 2002; 30:108-14.

24. Nadanovsky P, Sheiham A. Relative contribution of dental services to the change in caries level of 12year-old children in 18 industrialized countries in the 1970's and early 1980's. Community Dent Oral Epidemiol 1995; 23:331-9.

25. Antunes JLF, Narvai PC, Nugent ZJ. Measuring inequalities in the distribution of dental caries. Community Dent Oral Epidemiol 2004; 32:41-8.

26. Baldani MH, Vasconcelos AGG, Antunes JLF. Associação do índice CPO-D com indicadores sócioeconômicos e de provisão de serviços odontológicos no Estado do Paraná, Brasil. Cad Saúde Pública 2004; 20:143-52.

Recebido em 09/Abr/2008

Versão final reapresentada em 29/Out/2008

Aprovado em 18/Nov/2008 